\title{
Working Group in Model-based Design and Reasoning Part II: Design
}

\author{
Boi Faltings \\ Laboratoire d'Intelligence Artificielle, \\ Swiss Federal Institute of Technology (EPFL), \\ 1015 Lausanne, Switzerland, \\ faltings@lia.di.epfl.ch
}

\begin{abstract}
Conventional Artificial Intelligence techniques such as expert systems have often been found to be too brittle for large-scale applications. Model-based reasoning is a technique for making Artificial Intelligence software applicable to problems of realistic size.

In this working group, we have investigated some fundamental issues in model-based reasoning and various applications in diagnosis, control and design. This paper reports on the work at the Artificial Intelligence Laboratory, EPFL and the Chair for Computer-aided Architectural Design, ETHZ on applying model-based reasoning to design problems. The results concerning basic techniques and diagnosis applications are reported in another paper in this volume.
\end{abstract}




\section{INTRODUCTION}

Design is traditionally regarded as an activity where unstructured thought is of prime importance to allow innovation to arise. However, in the modern world, a designer has to consider an enormous amount of technical details which are hardly compatible with this requirement. Furthermore, design is now often carried out in a team where decisions must be shared with colleagues. Human intelligence is insufficient for this environment; designers require an artificial intelligence designed for problem-solving in highly structured and constrained domains.

Conventional CAD systems do not fulfill this goal: they are only drawing tools which provide no understanding and consequently no support for the design itself. Knowledge-based systems based rules, such as the ICAD system, are being used increasingly and successfully in large companies such as Boeing or General Motors. In this project, we investigate the use of modelbased reasoning to replace rule-based reasoning in intelligent design systems, for example in mechanical and building design.

The most straightforward way to apply models to design is by abductive reasoning where a structure is synthesized from components such that it fulfills a desired function. However, such abduction is computationally tractable only when components can be assigned functions in a way that is independent of their context of use (The no-function-in-structure principle). This condition is given for example in electronic circuits, in particular digital circuits, but not in domains which require a geometric layout, such as mechanisms and buildings. In such domains, the space of "good" designs is very sparse, and any particular component is likely to be useful only in very particular contexts (Figure 1). It is then more useful to model design knowledge as parameterized prototypes of complete designs. There exist several commercial tools for such parametric design.

Defining libraries of prototypes is a tedious process which only large companies can afford. In general, knowledge is available in the form of cases of earlier designs, and models of how they work. The novelty of our project is that we directly adapt cases to new problems, using models to define the admissible modifications.

To illustrate the approach, we have implemented two prototype systems:

- CADRE for case-based design of buildings, and 


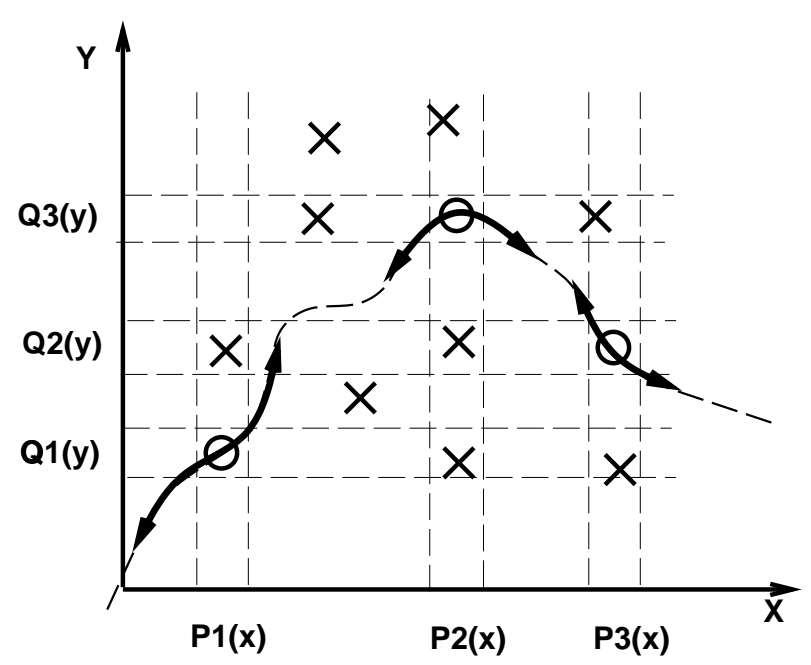

Figure 1: In highly context-dependent domains such as shape design for mechanism parts, the set of "good" designs (circles) among the "bad" designs (crosses) is often very sparse. Predicates P1-P3 and Q1-Q3 which distinguish good from bad often must be invented with a particular example in mind: P1-Q1, P2-Q3 and P3-Q2 cover the three positive examples shown here. In a prototype-based system, good designs are described as variations of prototypes, as indicated by the arrows. The space of "good" designs will often have a complex shape such as indicated by the dashed line, of which only the parts around the known prototypes are well-understood.

- FAMING for case-based design of part shapes in mechanisms.

Both systems allow innovation by case modification, combination and reinterpretation. They are tools for design support rather than automation. The tasks of identifying suitable cases and their interpretation are left to the user: they constitute the $1 \%$ inspiration which is hard to reproduce in a computer program. CADRE and FAMING then take over the remaining $99 \%$ perspiration by automatically proposing novel designs which implement the designer's inspiration.

Graphical interfaces are essential to make complex systems such as CADRE and FAMING usable by practicing designers. Researchers at ETH Zürich have developed a program called SCULPTOR for displaying and manipulating objects in virtual reality. While the program has not yet reached the level of 
complexity for handling actual design cases, it is intended to be integrated with CADRE in the future and will provide a powerful interface for designers using the techniques.

\section{INTELLIGENT OBJECTS}

Case-based reasoning is a technique where past solutions are reused or adapted to solve new problems. In case-based design, specific design precedents are reused for new problems. Because designs can never be reused exactly, a key issue in case-based design is how to adapt a case to a new problem. One can distinguish two types of adaptation:

- modification, where small changes are made to a single case until it fits the requirements, and

- combination, where two or more cases are combined into a new design.

For adaptation, it is crucial to know what changes can be made to a design case without perturbing its function. This knowledge, which we call adaptation knowledge, must be provided in addition to the model of the structure stored in the design case.

Our approach to provide adaptation knowledge for cases is to interpret them in terms of structure-behavior-function(SBF) models. Such an interpretation decribes a particular interesting function implemented by the structure. An SBF model defines how each element of the structure is responsible for aspects of the behavior, and how aspects of the behavior are in turn responsible for the function of the device. By associating an interpretation in the form of an SBF model with a case, it becomes an intelligent object which is capable of adapting itself to fit new specifications and environments.

Following common design methodologies such as that of Pahl \& Beitz, we assume functions and thus SBF models to be qualitative. A qualitative model differs from a quantitative one in that it specifies properties which hold over ranges of parameter values. This makes it possible to express functions which hold over a range of situations, such as "block any counterclockwise motion". Another reason for using qualitative models it that they make it possible to determine all possible behaviors of a device, not only a particular snapshot valid for certain input parameters. This is required for expressing certain specifications, such as "parts A and B should never loose contact." 


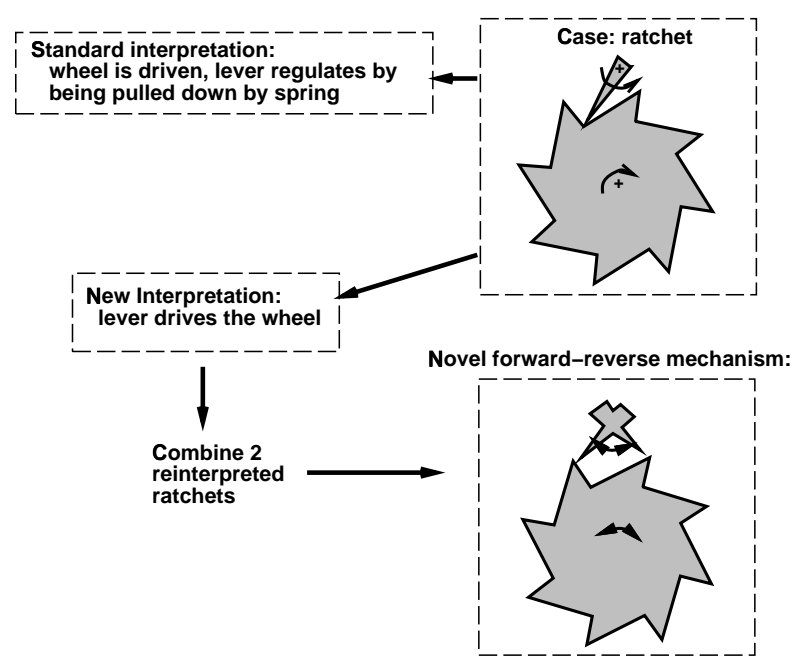

Figure 2: When viewed by a designer, artifacts are interpreted in terms of structure-behavior-function (SBF) models. Innovation often results from reinterpreting cases with different models than originally intended.

Innovation and creativity can arise in case-based design through adaptation and combination of cases which might yield very different results from what was previously known. Another powerful source of innovation is the reinterpretation of an existing case using a different SBF model. Figure 2 shows an example where a ratchet device is reinterpreted using a different SBF model than intended by the original designer. Combination of two such devices results in an innovative design of a forward-reverse-mechanism, a device which transforms an oscillating input motion into a rotation which advances two steps forward and one step backward with each oscillation (this example is described in detail in [2]). Such reinterpretation is the source of much innovation in design.

\section{FAMING: A CASE STUDY IN MECHA- NISM DESIGN}

FAMING $([1,2,3])$ is a system for supporting the creative design of elementary mechanisms, also called kinematic pairs. Examples of devices covered by 


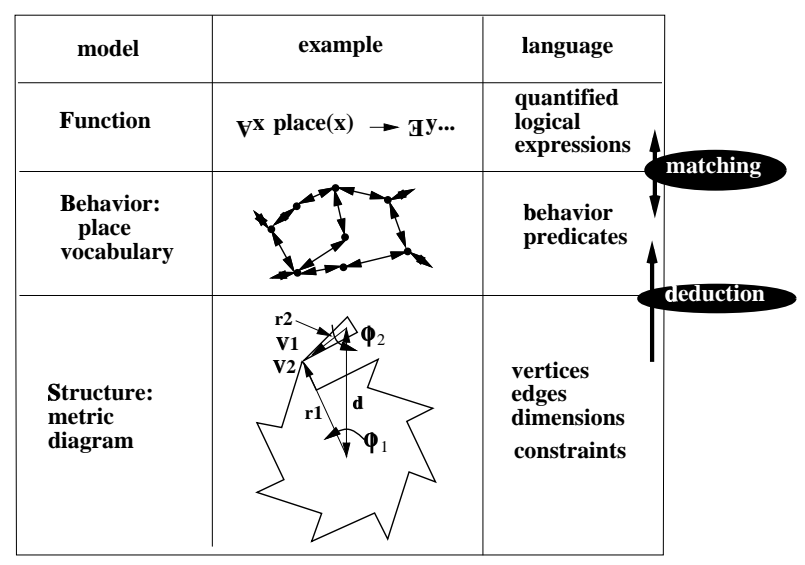

Figure 3: Representations of Structure, Behavior and Function models for mechanisms.

FAMING are ratchets and clock escapements.

\subsection{Models in FAMING}

Figure 3 shows the structures which implement the 3 models for structure, behavior and function in FAMING, and how they are related through reasoning processes.

\section{Structure: Metric Diagram}

The metric diagram is a CAD model which defines a kinematic pair as two two-dimensional objects consisting of vertices and edges between them. Each vertex defines two symbolic parameters; in the case of rotational freedom, an angle for its orientation and a scalar for its distance from the center of rotation of the corresponding object.

Based on the metric diagram, a shape feature (which may involve both objects) is defined by (i)a set of vertices and edges, and (ii) a set of constraints which must hold simultaneously for the shape feature to be present. For example, the shape feature which corresponds to the possibility of the top of the ratchet's lever being able to touch the wheel ( $v_{1}$ touching $v_{2}$ in Figure 3 ) can be expressed as follows: 
- must exist: vertices $v_{1}, v_{2}$

- constraints: $\left\{\left|d-r_{1}\right|<r_{2}\right\}$

\section{Qualitative Behavior: Place Vocabularies}

Textbooks on the subject explain kinematic behavior qualitatively by sequences of kinematic states. A kinematic state is defined by part motions and a contact relationship. For a kinematic pair, part motion is modelled qualitatively by a combination of signs which define the directions of each parts motion: $+, 0,-$, or ${ }^{*}$ which stands for any of the three. The set of possible contact relations depends on the part shapes. We call each different contact relation a place. For kinematic pairs, there are three different types of places: point-point or edge-edge contacts (zero-dimensional), point-edge contact (one-dimensional) and no contact (two-dimensional).

Depending on the place, only particular qualitative motions are possible, modelled by the predicate allowed-motion $(\mathrm{x}, \mathrm{d})$ where $\mathrm{d}$ is a vector of two signs. Motions can cause changes in contact relations. Places are arranged in a graph where each arc models a possible transition between contact relations. This is formalized by the predicate transition $(\mathrm{x}, \mathrm{y}, \mathrm{d})$, which is true whenever motion $\mathrm{d}$ can cause a transition from place $\mathrm{x}$ to $\mathrm{y}$.

\section{Function}

There has been much recent work on formalisms for representing function in design. Most researchers consider function to be a causal relation between an environment (or context) and a particular behavior, and are concerned mainly with vocabularies for specifying these causal connections.

In kinematics, there is only one form of causality, that of pushing on a part contact. In any particular state, the allowed-motion predicates allow predicting the behavior in an external environment and thus determine the function of the device. Often, functions are properties of sets of states, for example that there exists a certain sequence of states. We therefore formulate functions in two levels:

- a functional feature defines a property of a particular state or set of states, and thus always takes at least one state as an argument. 
- a device function defines a property of the entire behavior. It consists of a logical expression in functional features where all states are bound by quantifiers, and a specification of an environment assumed for this device function. We assume the environment to be independent of the mechanism state.

\subsection{Case Adaptation in FAMING}

For each case, FAMING takes as input a structural model of an existing device, represented as a metric diagram, and a qualitative model of its function, represented as a set of device functions. The first step in reusing the case consists of understanding how it implements the function, and consequently what can be changed without affecting this function.

Based on the information in the metric diagram, FAMING first computes the place vocabulary, which defines all possible behaviors of the device. These behaviors are then automatically matched to the specified device function. For example, assume that the functional specification of a device contains the condition that there exists a blocking-place $x$, defined by the fact that none of the externally imposed motions are allowed in place $x$.

Furthermore, assume that the place vocabulary of the corresponding case contains a place $P$ which satisfies this criterion. Unification of the functional specification with the place vocabulary substitutes $P$ for $x$, thus transforming $(\exists x)$ blocking-place $(\mathrm{x})$ into blocking-place(P). Assume that the device environment allows the qualitative motions $\{(-,-),(-, 0),(-,+)\}$; these are thus the motions which must be blocked in $P$. Replacing the blocking-place predicate by the full expression in its definition and expanding the quantification over all motions, we obtain the following conjunction of predicates:

$$
\begin{aligned}
& \neg \text { allowed }-\operatorname{motion}(P,(-,-)) \wedge \\
& \neg \text { allowed }-\operatorname{motion}(P,(-, 0)) \wedge \\
& \neg \text { allowed }-\operatorname{motion}(P,(-,+))
\end{aligned}
$$

Next, FAMING translates this understanding into the shape features which are responsible for implementing the device function. Each predicate in the conjunction is implemented by particular aspects of the object shapes. Using 


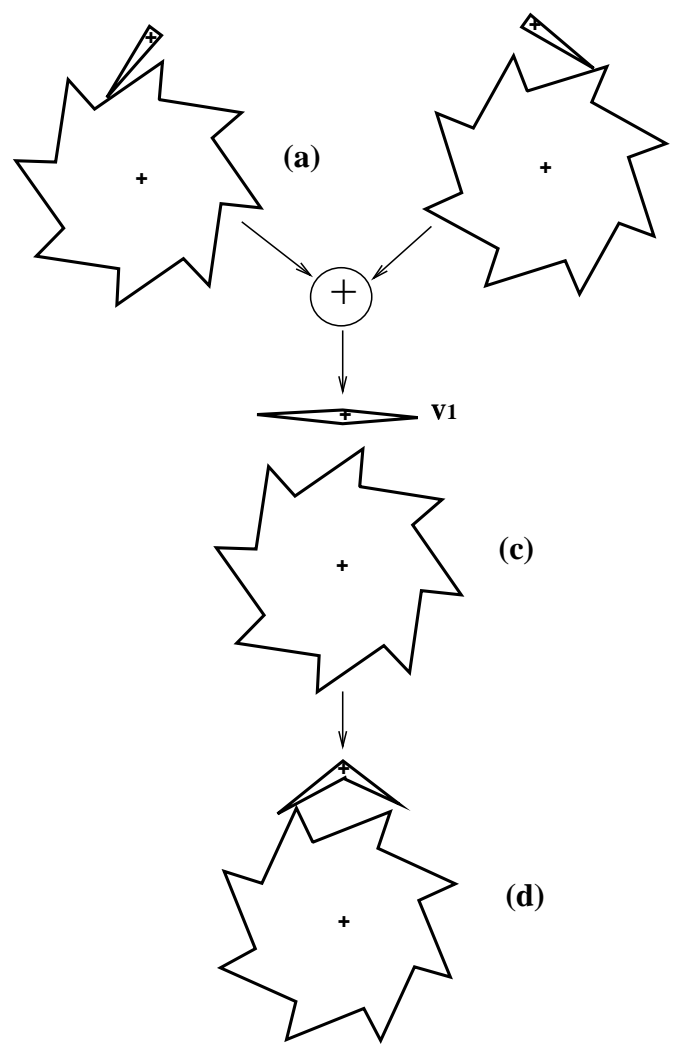

(b)

Figure 4: Design of an escapement by combining two ratchet devices.

a trace of their computation, it is possible to determine the constraints under which the behavior predicates remain valid. The behavior predicate $\neg$ allowed $-\operatorname{motion}(P,(-,-))$ can be mapped into constraints on the positions of object vertices. FAMING provides such mappings for all kinematic properties modelled in the place vocabulary. Reversing the causal chain of the analysis thus establishes a mapping from functional features to shape features, and we call such a process structure-behavior inversion. More details on the mapping between shape and qualitative behavior can be found in [3] 


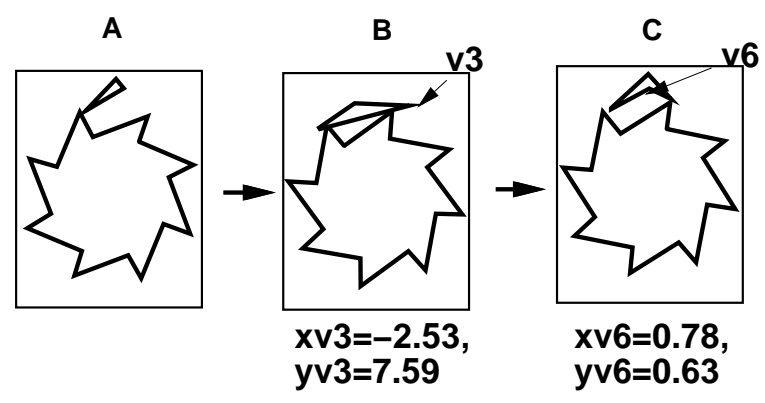

Figure 5: Incremental design of an escapement using modification operators derived by causal inversion. The program first changes the position of vertex $v 3$, and after discovering further constraints introduces vertex v6 to make the constraint system satisfiable.

\subsection{Examples of FAMING}

Being a research program, FAMING only treats devices from a limited domain, namely two-dimensional fixed-axis kinematic pairs with polygonal part shapes. This domain covers a large set of practical mechanisms, such as ratchets or escapements. The program has been used to design an escapement both by composing two ratchet devices (Figure 4) and by incremental modification of a single ratchet (Figure 5 .

FAMING has also been used to design entirely novel devices and thus shown its potential for creativity. In [2], we show how reinterpretation of a ratchet structure and combination of two reinterpreted devices are used to create a novel forward-reverse mechanism which is much simpler than the solution proposed in the literature.

\section{CADRE: A CASE STUDY IN ARCHITE- CTURE}

In earlier work, we have developed a prototype system for constraint-based case adaptation of buildings. In the context of this project, Kefeng Hua of the EPFL has extended this prototype into the CADRE system $([4,6])$. Figure 6 shows the structure of CADRE. 


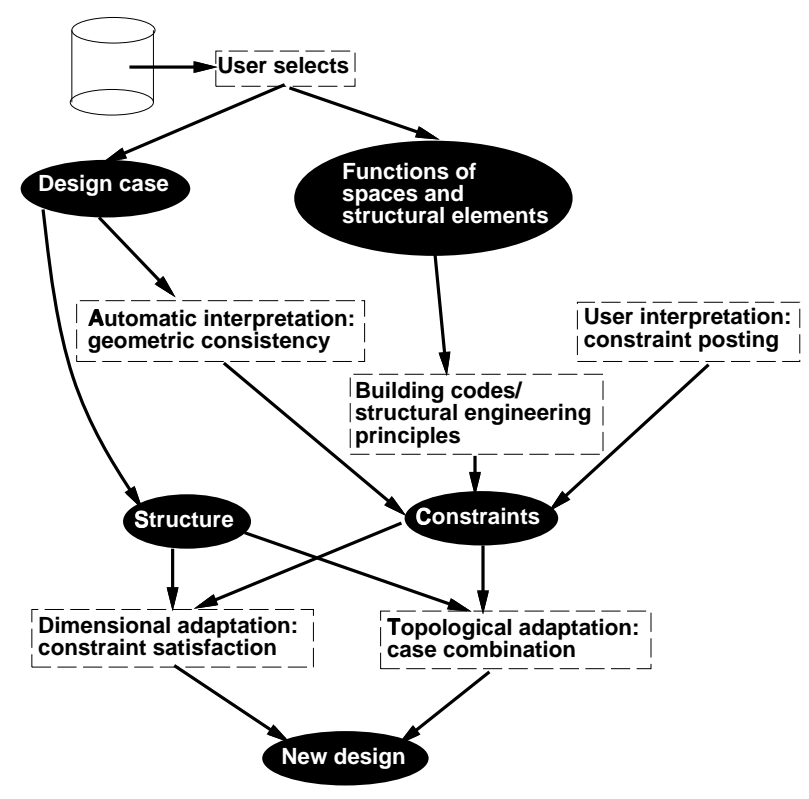

Figure 6: Data flow and processes in CADRE

\subsection{Structure, Behavior and Function in CADRE}

In CADRE, cases are represented as AutoCAD models of existing buildings or parts of buildings. Interpretations of these cases again consist of three components: structure, behavior and function, but due to the domain their separation is less clear than in FAMING.

\section{Structure models}

The AutoCAD model defines a structural model consisting of:

- objects, i.e. the vertices, lines and planes of the AutoCAD model.

- variables for the positions or dimensions of these objects.

- a set of values currently assigned to the variables.

This is similar to the metric diagram in FAMING. 


\section{Behavior and Functional models}

Since buildings do not normally change over time, their function and behavior cannot be understood in terms of a temporal evolution. The corresponding concepts in buildings are defined by the use that occupants make of the space. For example, a space can be used as an office by putting desks and chairs in it, or as a kitchen by installing a stove and a sink, and this defines its function. The space then manifests a certain behavior: for example, if there is not enough space in an office to access the desk, or not enough light to work at it, then the "office" behavior is not satisfied.

To avoid the complexity of modeling behavior at this level of detail, in CADRE all this is modelled by constraints formulated directly on the structure. This in fact corresponds to current practice in the building industry, as building codes fix constraints for each particular use to ensure that the behavior of the spaces makes this use possible. Constraints can be instantiated automatically from a library by assigning functions to spaces in the case.

\subsection{Intelligent objects in CADRE}

We formulate cases as instances of particular buildings along with a set of constraints which restrict the possible modifications which can be made to them. We distinguish two forms of adaptation:

- dimensional adaptation, where only dimensions of the case are changed, and

- topological adaptation, where the arrangement and number of spaces and walls are also modified.

We have developed a general solution to this problem for dimensional adaptation, based on the notions of dimensionality expansion to make conflicts resolvable and subsequent dimensionality reduction to limit the complexity of modifications.

In some cases, it is not possible to adapt a case to a new environment using only changes to its dimensions; its topology has to be modified as well. Certain structures can be varied in a regular manner: for example, a string of rooms can be shortened by adding or removing one of them. However, in 

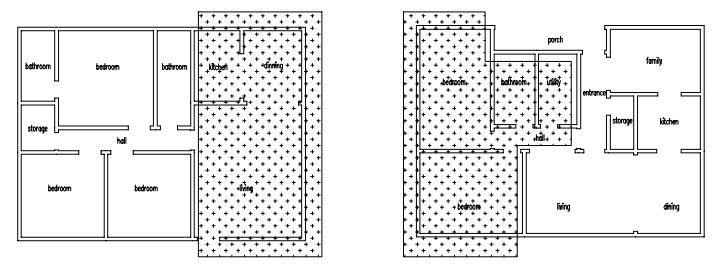

Figure 7: The unshaded parts show case fragments to be combined and adapted by CADRE.

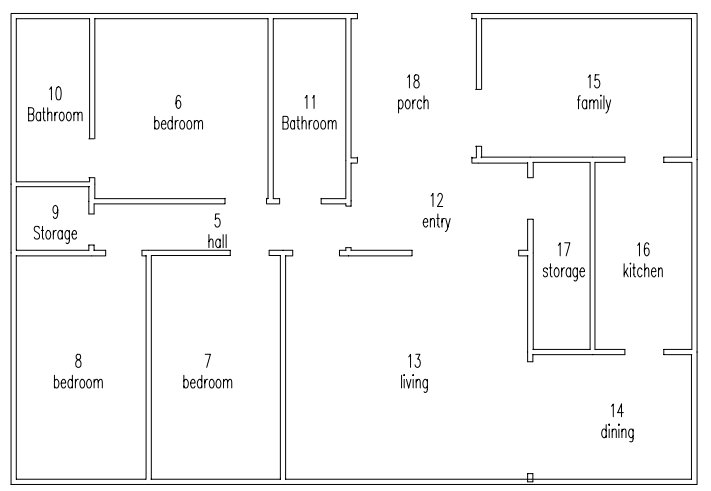

Figure 8: Result of adaptation by CADRE.

most cases a topological change is likely to destroy the qualities the user sees in the case. The principle in CADRE is therefore that changes in topology are allowed only when the new topology can be found in another case. Significant parts of the new case are selected at the appropriate level of abstraction and combined with the existing one. The details of the process are described in $[5]$.

Our prototype system, CADRE, has been tested on examples of realistic complexity. Figures 7 and 8 show an example of an adaptation of a layout carried out by the program. The user has specified the combination of the non-shaded parts of the two shown cases. The case on the left is called the host case, meaning that it dominates in case of conflicts, and the case on the right is a guest case which is adapted to fit the host case. One of the solutions proposed by CADRE using both dimensional and topological modifications is shown in Figure 8. 


\section{CONSTRAINT SATISFACTION TECHNI- QUES}

Both CADRE and FAMING translate case interpretations into constraints on the structures. This defines a constraint satisfaction problem (CSP) whose solution is the adaptation of the case which respects the interpretation that was attached to it. This constraint satisfaction problem involves many constraints on continuous variables, and there are no general and reliable methods for solving it available in the literature. Iterative refinement methods such as "blackboard" structures suffer from infinite cycling problems and are not guaranteed to ever find a solution. Optimization techniques in operations research make many implicit assumptions about the shape of the solution spaces which are often not satisfied in design.

For the purpose of the techniques we describe here, we have developed a comprehensive framework that allows efficient and complete solution of the constraint networks encountered in case adaptation. It relies on two concepts:

- equality constraints are subjected to a process called dimensionality reduction: in each constraint, one parameter becomes a dependent parameter which cannot be chosen during adaptation. It is thus eliminated from consideration: the dimensionality of the prolblem is reduced.

- in the remaining system of inequalities, all parameters eliminated during dimensionality reduction are replaced by the expression obtained through solving the equality. The resulting system is then solved using a novel propagation algorithm developed in this project and described below.

Dimensionality reduction is implemented using Gaussian elimination for linear constraints, the MAPLE software package for certain nonlinear constraints, and special heuristics for other types of nonlinear constraints. This is described in detail in [5].

After dimensionality reduction, the task is to solve a system containing only inequalities. We solve such systems in two steps:

1. we find the consistent solution regions, and

2. we instantiate particular solutions within the regions, either automatically or through user interaction. 


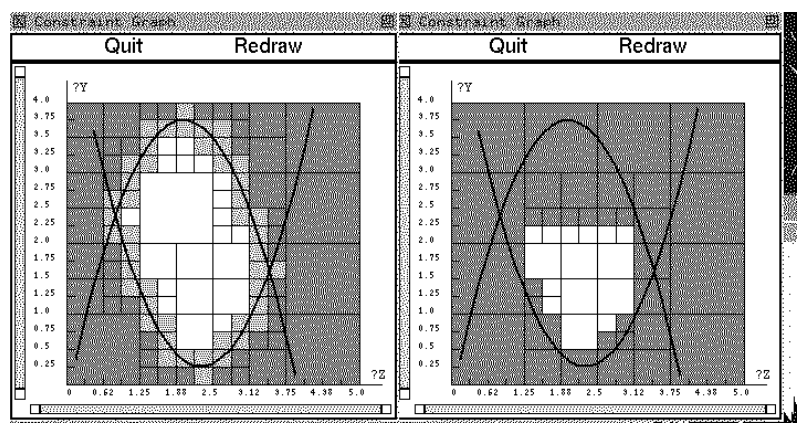

Figure 9: Example of the quadtree decomposition constructed for a set of constraints (left), and the feasible regions (shown in white) after computing global consistency with the rest of the constraint network (right).

Existing propagation techniques for solving systems of continuous variables are unsatisfactory in several respects:

1. known local propagation rules fail to achieve the desired arc-consistent result in many practical cases.

2. propagation rules based on interval bounds fail to terminate.

3. many practical constraint networks contain cycles and require propagation algorithms which achieve more than local consistency.

Problem (1) is due to the fact that multiple constraints between the same pair of variables are propagated individually. In [7], I show that arc-consistency is guaranteed when constraints are propagated as a single total constraint, and give a simple algorithm for carrying out such propagation. The method is defined for binary constraints only. It can be extended to multiple variables in a straightforward manner.

Local propagation of interval bounds in constraint networks with cycles amounts to solving a system of simultaneous equations. Propagating constraints individually means that the system is solved by a numerically unstable fixed-point iteration, and can cause infinite cycling. A method which is both reliable and guaranteed to converge rapidly on such problems is that of binary search. Binary search recursively computes finer and finer approximations of the solution. 
Djamila Haroud of the EPFL Artificial Intelligence Laboratory has developed a method for propagating total constraints (as shown necessary in [7]) using such a recursive decomposition ([8]). Total constraints between any pair of variables are approximated using a quadtree decomposition, similar to that used in computer vision (see Figure 9). The minimum granularity of the approximation determines the precision with which intervals of feasible variable values will be computed and is determined by the requirements of the application. It can be shown that local consistency algorithms based on this approximation always converge rapidly even when the constraint network contains cycles.

Local consistency is not always enough for solving design problems. Computing higher degrees of consistency results in exponential time complexity and is not feasible in practice. However, research on the temporal reasoning problem has shown that in cases where constraints only admit single convex intervals, path-consistency (which can be computed in low-order polynomial time) is equivalent to global consistency. We have generalized this idea to general constraint satisfaction problems where total constraints are unions of convex regions, and thus obtained an algorithm which determines globally consistent solutions for many classes of continuous CSPs in polynomial time $([8])$.

\section{INTELLIGENT OBJECTS IN VIRTUAL REALITY}

The artificial intelligence provided by systems such as FAMING and CADRE must be connected to the natural intelligence of the human designer in a smooth manner. Design is concerned with three-dimensional objects which are very tedious to manipulate using common two-dimensional graphical interfaces. This makes the systems exceedingly complex to use in pratical applications.

David Kurmann and Gerhard Schmitt of the chair for computer-aided architectural design (CAAD) of the ETH Zürich have developed a program called SCULPTOR ([10,11]) which provides a three-dimensional, virtural reality interface allowing a designer to manipulate objects in an intelligent design system. 
In SCULPTOR, users perceive objects in a virtual design environment created through stereo images and sound. They can change objects and their arrangement using direct manipulation with a three-dimensional mouse, a great simplification over current CAD systems which require manipulation of several viewplanes. When a case is adapted interactively by the designer, SCULPTOR enforces the constraints defined by the FBS models by giving the objects certain behaviors. For example, if the size of a room cannot be reduced below a certain limit, the corresponding object will be deformable only up to this limit.

In order to maintain the illusion of virtual reality, constraints have to be maintained in real-time. This limits the complexity of constraint processing that can be permitted. Kefeng Hua of the EPFL has implemented a simplified version of CADRE, dealing only with floor layouts. Due to this simplification, constraint management is sufficiently fast and this simplified program has been perfectly integrated with SCULPTOR. We are now investigating techniques which allow the more time-consuming constraint management processes associated with complex design problems to be decoupled from real-time manipulation to allow the integration of more complex design systems with SCULPTOR.

Adaptation often involves a search among many alternative solutions. Rather than search on their own initiative, modern users prefer to let the computer perform the search and only select the most satisfactory result. For this purpose, SCULPTOR provides a functionality where the user can set objects in motion independently and watch their movement in space ([9]). When several objects are moving independently and respecting the constraints, this corresponds to a systematic search of arrangements possible within the constraints. When a pleasing configuration is reached, the user can stop the motion and continue working with the result.

\section{CONCLUSIONS}

Human intelligence has evolved to help people survive in natural environments: it is very good at handling natural, rounded shapes, reasoning with approximate information, and similar tasks. Modern engineering operates in a highly constrained world of artificial shapes with sharp edges, precise and well-controlled interactions, and many constraints to be respected. Human 
intuition is not well-suited to this environment, and its creativity cannot be exploited to its fullest.

By automating the low-level details, FAMING provides the designer with a "mechanical intuition" which he does not normally have and which makes it much easier to him to create novel devices. Using its numerical precision, the program provides him with the capability to envision and thus design devices whose behavior depends on precise shapes and interactions.

In building design, architects often produce designs which are costly or even expensive to build. Even worse, in complex design projects there are many inconsistencies which are only discovered late in the construction process and cause tremendous cost overruns. A tool such as CADRE frees an architect from having to worry about low-level details ranging from geometric consistency of drawings to the feasibility of integrating a suitable structure. He can now concentrate on composing spaces to implement the function he desires.

The integration of SCULPTOR and CADRE shows an interesting synergy between artificial intelligence and advanced computer graphics. On the one hand, the increased interaction bandwith made possible by virtual reality makes it possible for users to really integrate the "intelligence of the artificial" provided by the computer with their own perception of form and style: virtual reality is needed to make knowledge-based systems useful. On the other hand, in order to be convincing, virtual reality systems require complex models of behavior which can only be created using large amounts of knowledge: knowledge-based systems are required to make virtual reality useful. Further interesting synergies can be expected from the combination of the two technologies.

\section{Acknowledgement}

The author would like to acknowledge the following members of the SPP project No. 5003-034269 for their contributions reported in this paper: Djamila Haroud, Kefeng Hua, Ian Smith and Kun Sun of the EPFL Artificial Intelligence Laboratory; David Kurmann and Gerhard Schmitt of the Chair for Computer-aided Architectural Design at the ETHZ. The work on CADRE built on some of the results obtained in a project carried out under the national research program 23 on Artificial Intelligence and Robotics. 


\section{References}

[1] B. Faltings, K. Sun: "Computer-aided Creative Mechanism Design," Proceedings of the IFIP International Conference on Feature Modeling in Advanced CAD/CAM Systems, Valenciennes, France, May 1994

[2] K. Sun, B. Faltings: "Supporting Creative Mechanical Design," in J. Gero, F. Sudweeks(eds.): Artificial Intelligence in Design '94, Kluwer Academic Publishers, 1994

[3] K. Sun: "Computer-aided Creative Mechanism Design," Ph. D. Thesis, EPFL, August 1994

[4] K. Hua, B. Faltings: "Exploring Case-based Building Design CADRE," Artificial Intelligence in Engineering Design, Analysis and Manufacturing 7(2), pp. 135-143, 1993

[5] K. Hua: "Case-based Design of Geometric Structure," Ph. D. Thesis, EPFL, August 1994

[6] I. Smith, B. Faltings: "Spatial Design of Complex Artifacts using Cases," Proceedings of the 10th International Conference on Artificial Intelligence for Applications, IEEE, 1994

[7] Boi Faltings: "Arc-consistency for continuous variables," Artificial Intelligence 65, 1994

[8] D. Haroud, B. Faltings: "Global Consistency for Continuous Constraints," Proceedings of the 11th European Conference on Artificial Intelligence, Wiley, 1994

[9] F. Wenz, D. Kurmann: "ImPuls", in G. Hartwagner, S. Iglhaut, F. Rötzer (eds.): Künstliche Spiele, Boer, Munich, 1993

[10] G. Schmitt, F. Wenz, D. Kurmann: "Die kurze physische Präsenz der Architektur," Ars Electronica, Linz, June 1994

[11] G. Schmitt: "Scene Animation using Intelligent Objects," Speedup, 1994 